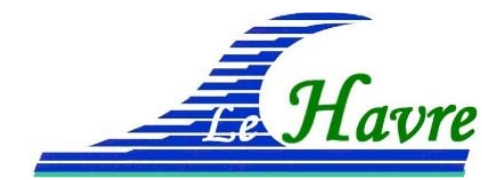

XVI èmes Journées Nationales Génie Côtier - Génie Civil

Le Havre, 2020

DOI:10.5150/jngcgc.2020.023 C Editions Paralia CFL

disponible en ligne - http://www.paralia.fr - available online

\title{
Modélisation de la dynamique sédimentaire au niveau du port de Cap Djinet, Algérie
}

\section{Hocine DAHMANI ${ }^{1,2}$, Fouzia HOUMA BACHARI ${ }^{1,2}$, Cherif AOUDJ ${ }^{1,2}$}

1. Ecole Nationale Supérieure des Sciences de la Mer et de l'Aménagement du Littoral, ENSSMAL, Bois des Cars, B.P. 19 Dely Ibrahim, 16320, Algérie. hocinedahm@yahoo.fr

2. Laboratoire de l'Écosystème Marin et Littoral, EcoSys, Chemin du complexe de Sidi Fredj, Staoueli 16096, Algérie.

\section{Résumé :}

Les processus hydrauliques, et sédimentaires dans les zones côtières sont dus aux effets de la marée, du vent, de la houle et des courants qu'ils produisent (VIGUIER, 2002). Au cours de la construction du port de Cap Djinet démarrée en 2006, il a été constaté l'apparition du phénomène d'engraissement de part et d'autre des ouvrages de protection du port surtout au niveau des jetées. L'aspect le plus spectaculaire est visible au niveau de l'enracinement de la jetée principale dont le tronçon constituant son enracinement, a vu sa carapace recouverte par un important volume de sable. La flèche sableuse créée à l'abri de la jetée principale atteignant le niveau zéro, témoigne également de l'ampleur de ce phénomène d'ensablement. En vue de cerner la problématique posée et d'identifier les facteurs régissant ce phénomène ainsi que son origine, une étude a été faite qui recourt à la simulation numérique en utilisant un système de calcul puissant dénommé MIKE 21 . Ce système de calcul nous a permis de représenter la dynamique hydro-sédimentaire de la zone d'étude en l'absence d'ouvrage de protection (état initial) et de tester l'efficacité d'un certain nombre de diapositifs de lutte contre l'ensablement et de proposer par conséquent une solution qui réponde aux critères techniques et économiques.

Mots clés :

Sédimentologie, Houle, Courant, Hydrodynamique, Modélisation numérique, Mike21, ArcGis

\section{Introduction}

Le port de Cap Djinet se situe à environ $75 \mathrm{~km}$ à l'Est d'Alger et à moins de $20 \mathrm{~km}$ à l'ouest de la ville de Dellys (voir figure 1). Il est protégé par deux jetées, la principale au nord et la secondaire au sud de la passe d'entrée. Tout au long de la côte se développe une plage de forme allongée qui s'étale de l'embouchure de l'Oued Isser à l'Oued Sébaou. Cette zone littorale est également caractérisée par un transit littoral des sédiments alimenté par les oueds précités, principalement l'oued Isser qui a un débit important pour une crue centennale ( $\mathrm{Q}_{100 a n s}$ plus de $3000,0 \mathrm{~m}^{3} / \mathrm{s}$ ). La côte de Cap Djinet (au sud du port) a connu dans les années 90 une érosion remarquable de la plage menaçant par endroits 


\section{Thème 2 - Dynamique sédimentaire}

l'intégrité de la route nationale RN 24. Une étude de protection du site a été élaborée en 1992. La solution de protection du rivage recommandait la mise en place de deux épis et un brise-lames, mais la côte a eu de nombreux problèmes d'érosion localisés surtout du côté Sud-Ouest du site. Des travaux de protection ont été entrepris localement par la mise en place de murs de soutènement en gabions et en béton.

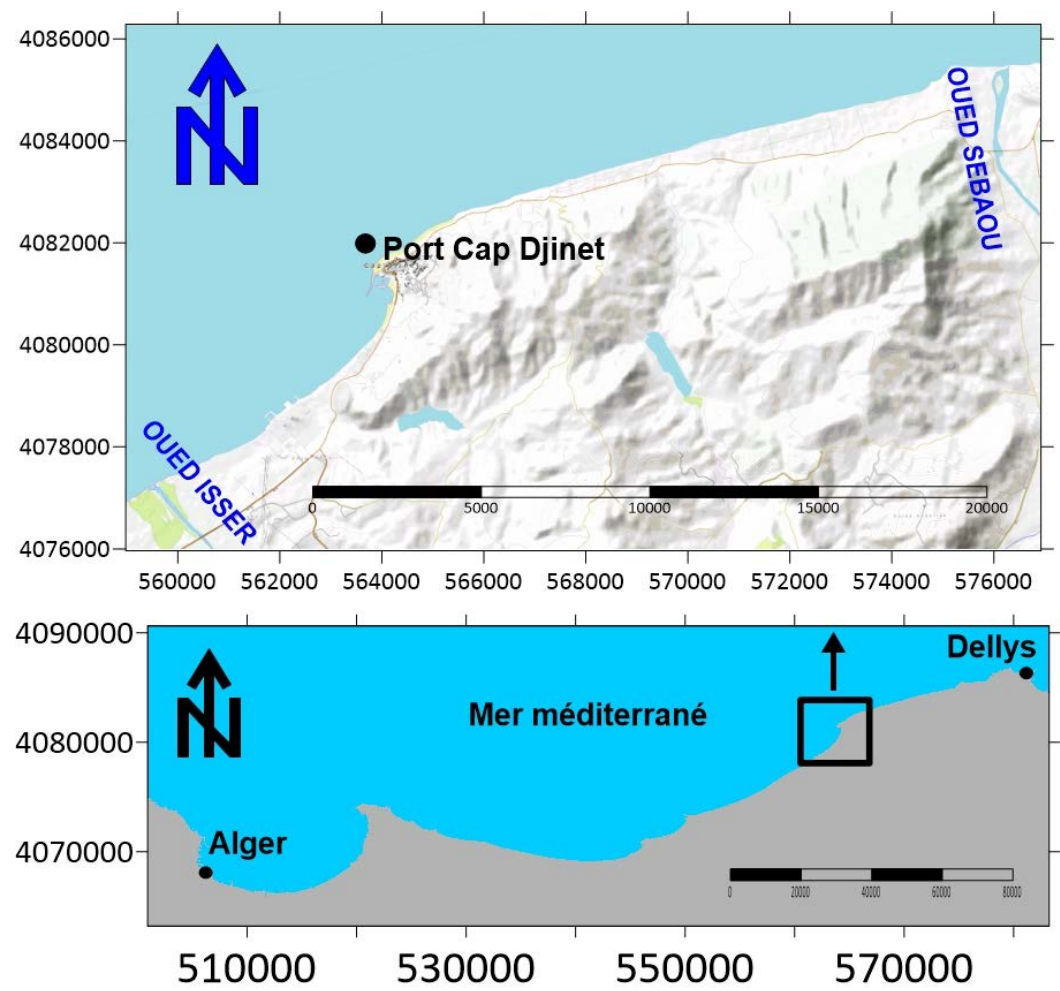

Figure 1. Carte de situation de la zone d'étude, port de Cap Djinet.

$\mathrm{Au}$ cours des deux années de construction du port 2006-2008, d'importantes modifications sur le plan bathymétrique sous forme d'une sédimentation apparente au nord et au sud du port ainsi qu'au niveau de la passe d'entrée ont été notées. Ce processus a atteint des proportions incompatibles avec l'exploitation du port. La carte (voir figure 2) illustre l'évolution du trait de côte pendant 10 ans (2009/2019) au sud du port ce que confirme un important transport sédimentaire dans la zone d'étude. La présente étude aura examiné la problématique posée par une approche numérique afin d'analyser les principaux facteurs océanographiques et sédimentologiques, le diamètre du grain médian (D50) est compris entre $110 \mu \mathrm{m}$ à $250 \mu \mathrm{m}$ (BOUHMADOUCHE, 2012). La mise en évidence et la compréhension des phénomènes régis par ces facteurs nous permettront de mieux cerner le problème et de définir les moyens efficaces et appropriés ainsi que les dispositions à prendre en considération pour remédier au problème posé. A ce propos, il est primordial de prévoir l'évolution du transit sédimentaire notamment la détection des zones d'érosion et de dépôt au niveau du rivage (MIHOUBI et al., 2011). Il est bien 


\section{XVIèmes Journées Nationales Génie Côtier - Génie Civil \\ Le Havre 2020}

évident, que certaines modifications des zones côtières sont liées à l'action anthropique, telles que la réalisation des aménagements hydrotechniques sans tenir compte du régime marin et des interactions avec les différents facteurs énumérés précédemment (MIHOUBI et al., 2012).

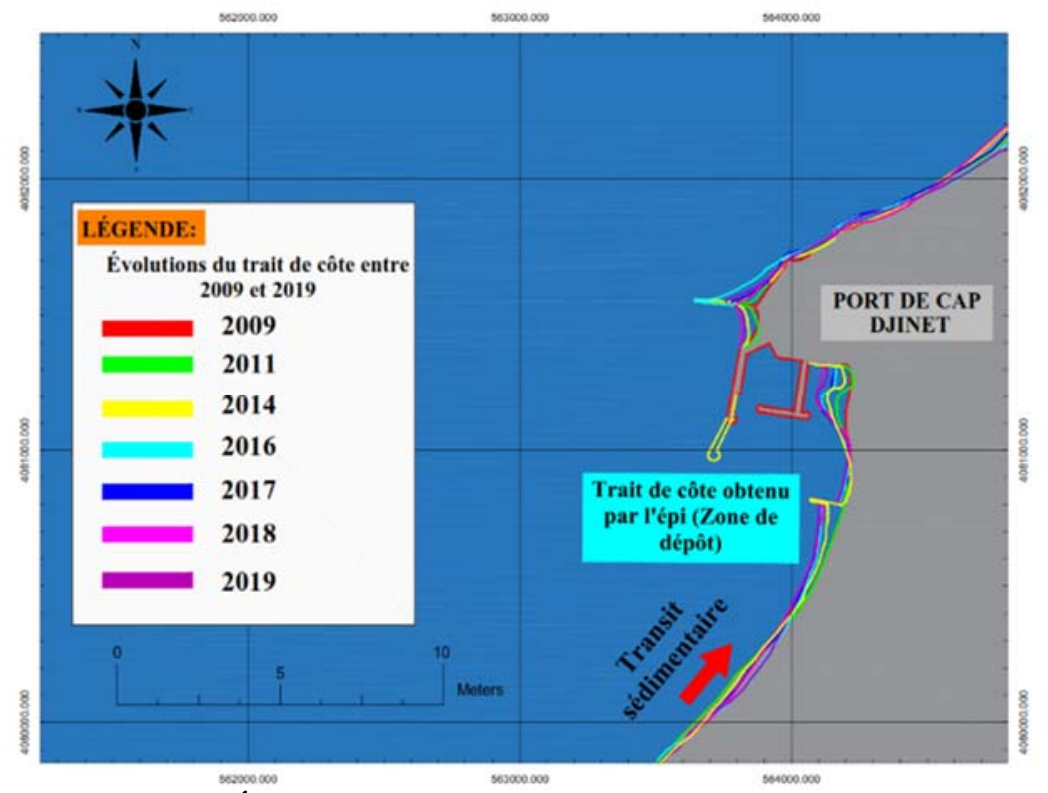

Figure 2. Évolutions du trait de côte entre 2009 et 2019.

\section{Modélisation hydro sédimentaire}

\subsection{Description du modèle numérique MIKE21}

La modélisation numérique est basée sur des modèles mathématiques décrivant de manière suffisamment fiable les phénomènes physiques. Le recours à une telle modélisation constitue une approche pratique vu qu'elle permet de tester les effets d'un grand nombre de paramètres (physiques et géométriques) en un temps relativement court et à moindre coût comparativement à un modèle physique équivalent. Le modèle numérique utilisé dans le cadre de cette étude est MIKE 21 qui est un puissant système de calcul (DAHMANI, 2012). L'approche méthodologique de modélisation intégrée du transport de sédiments non cohésifs par le code MIKE 21 est présentée à la figure 3. 


\section{Thème 2 - Dynamique sédimentaire}

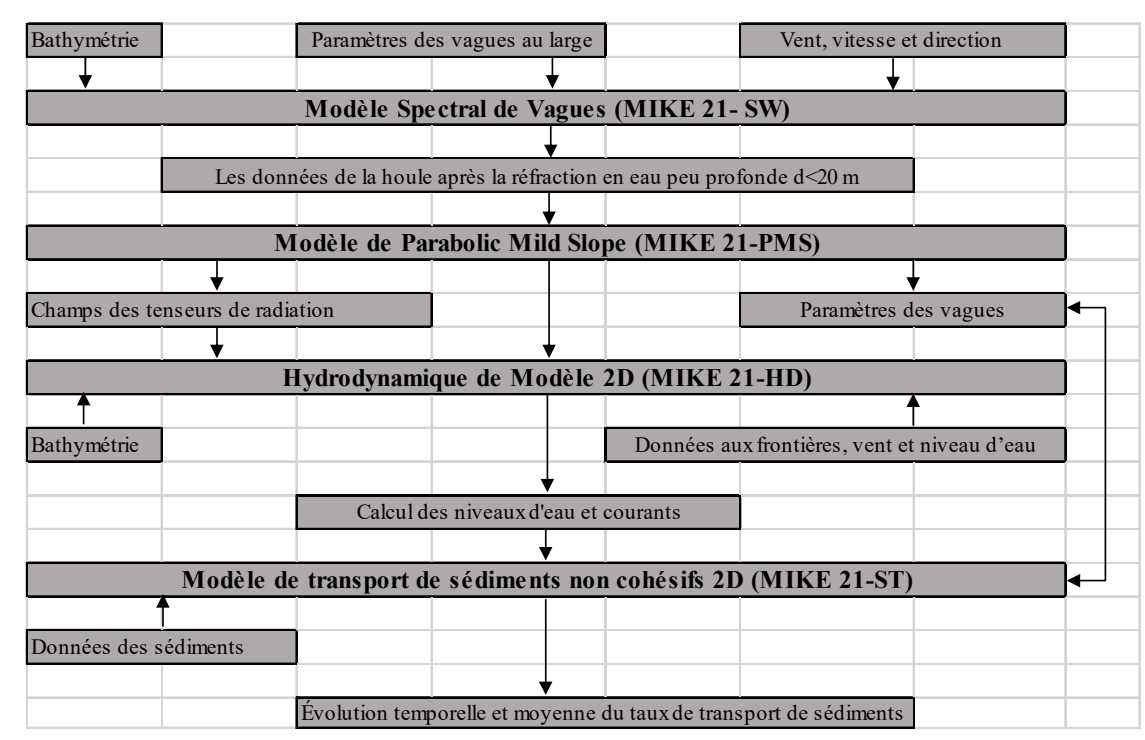

Figure 3. Approche méthodologique de modélisation intégrée du transport de sédiments non cohésifs par le code MIKE 21.

2.2 Simulation numérique du transit sédimentaire du port Cap Djinet

Dans le but d'identifier les facteurs responsables de l'ensablement du port de Cap Djinet, nous avons simulé les phénomènes physiques du site d'étude sur la base du levé bathymétrique réalisé dans le cadre de cette étude en 2019 (voir figure 4), ainsi que sur les données bathymétriques issues de la digitalisation de la carte marine du SHOM.

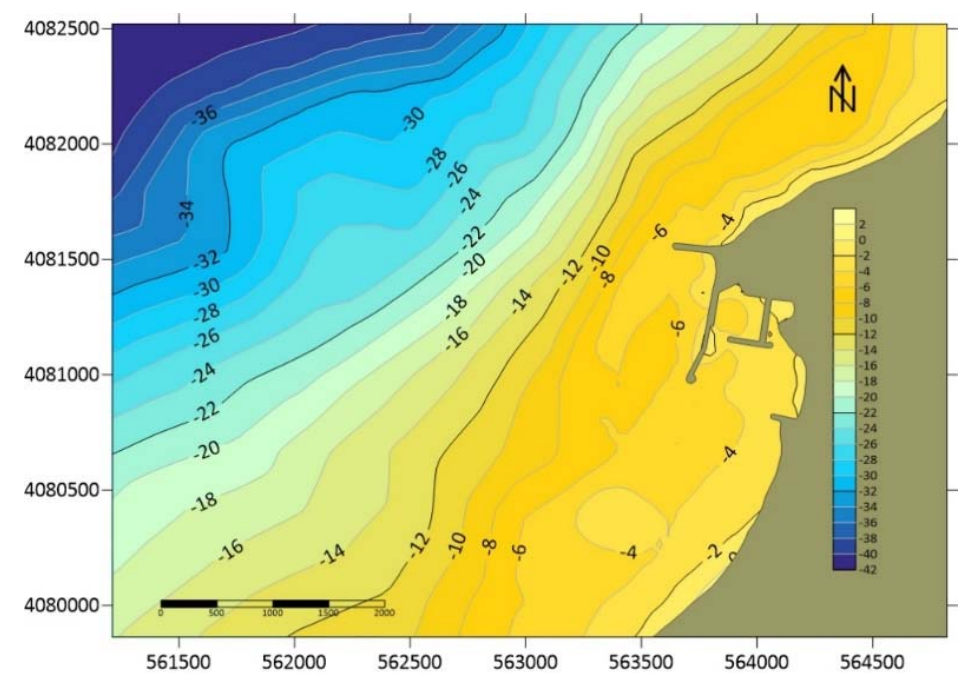

Figure 4. Levé bathymétrique de la zone d'étude port Cap Djinet.

Le domaine de calcul représentant la zone d'étude délimite une aire importante $(3.60 \mathrm{~km}$ $\mathrm{X} 2.65 \mathrm{~km}$ ) afin que les conditions aux limites soient en accord avec les phénomènes physiques en présence (caractéristiques de la houle au large et les courants littoraux induits). Pour la génération des simulations, nous avons choisi les quatre (04) classes de 


\section{XVİ̀mes Journées Nationales Génie Côtier - Génie Civil \\ Le Havre 2020}

houles les plus fréquentes et les plus importantes, leurs effets étant prédominants. Ces houles découlent des observations de navire entre 1963 et 1970 par l'US Naval Weather Command (MIHOUBI \& DAHMANI, 2015). Cette étude consiste à restituer successivement le champ de la houle, les courants induits, la capacité théorique de transport solide pour les directions $45^{\circ} \mathrm{N}, 360^{\circ} \mathrm{N}, 315^{\circ} \mathrm{N}$ et $270^{\circ} \mathrm{N}$. Les simulations numériques sont réalisées pour une durée d'action de six (06) heures qui correspondent au temps nécessaire à l'équilibre hydrodynamique du modèle numérique.

\section{Résultats et discussion}

\subsection{Etat initial}

Après avoir examiné le site et la mise en évidence des facteurs hydrodynamiques à l'origine de l'ensablement du port, il est clair que les courants littoraux constituent la force motrice du transit sédimentaire suivant le sens dominant des houles incidentes dans la direction Nord Sud, c'est-à-dire en direction du port. Les résultats de la concentration maximale du taux de transport de sédiments en fonction de la direction de la houle ont été reportés sur le diagramme ci-dessous (voir figure 5). Selon ce diagramme, les directions $315^{\circ} \mathrm{N}, 270^{\circ} \mathrm{N}$ et $360^{\circ} \mathrm{N}$ sont les plus fréquentes et les plus énergétiques. Dans cette communication, il est présenté les résultats pour la direction dominante $\mathrm{MWD}=315^{\circ} / \mathrm{Hs}=2.64 \mathrm{~m} / \mathrm{T}=8.0 \mathrm{~s}$.

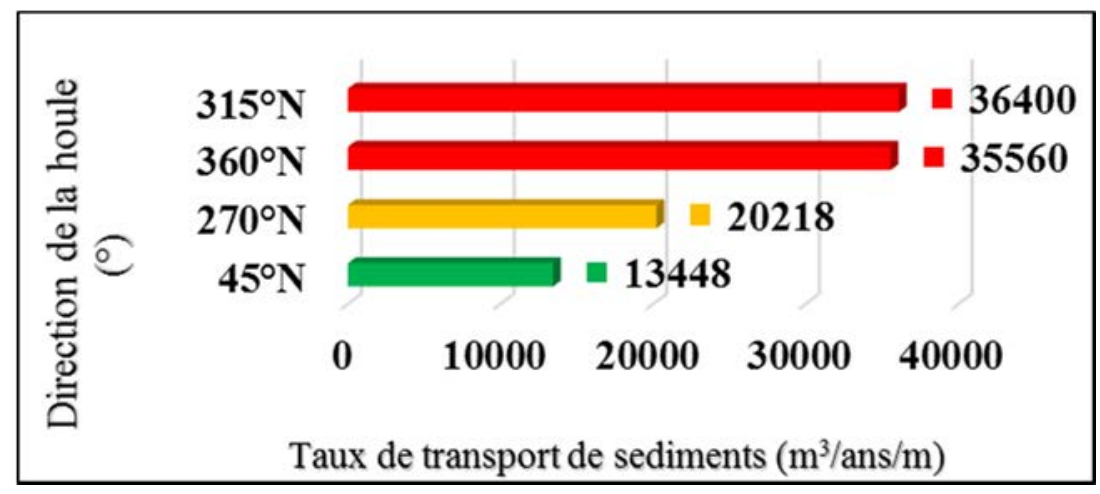

Figure 5. Concentration maximale du taux de transport de sédiments en fonction de la direction de la houle.

La figure 6 montre la bathymétrie du modèle sous forme d'un maillage avec 1'extension d'un format « .mesh ». La propagation de la houle (voir figure 7) se traduit également à l'approche du rivage par une modification de ses caractéristiques, à savoir une diminution de la hauteur significative de la houle et une déviation de l'angle d'incidence (phénomène de réfraction). On note également la diffraction au niveau du musoir de la jetée principale (voir figure 8). En s'approchant du rivage, les contraintes de radiations sont en évolution décroissantes selon la figure 9. 
Thème 2 - Dynamique sédimentaire

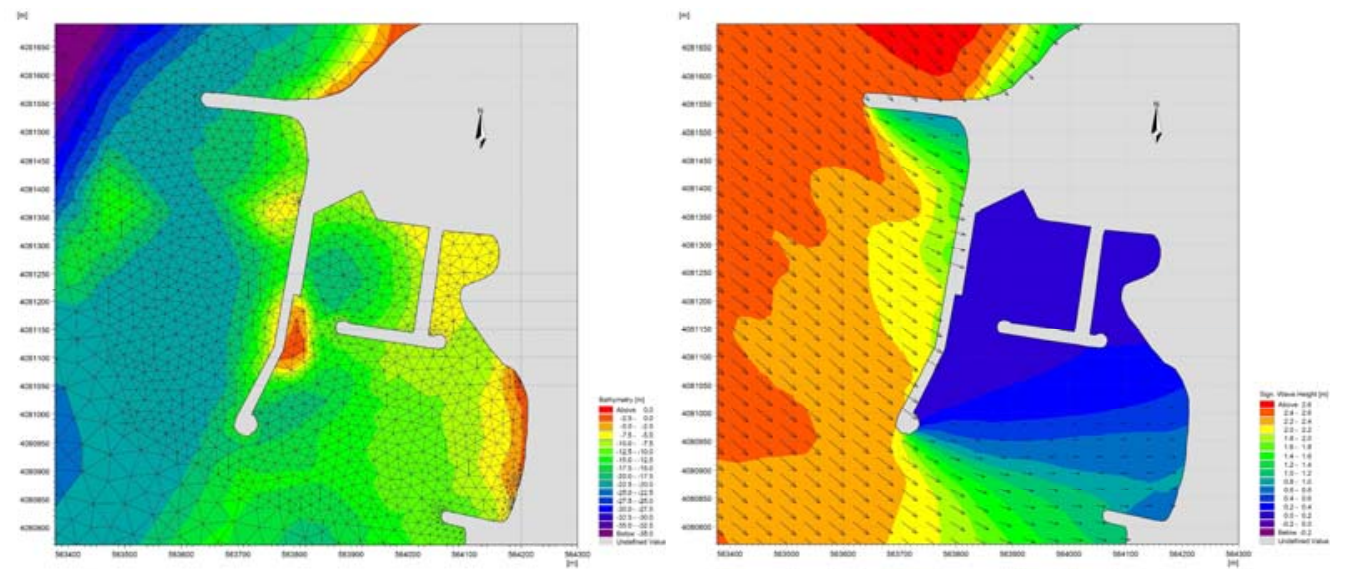

Figure 6. Maillage de levé bathymétrique. Figure 7. Caractéristique de la houle au large.
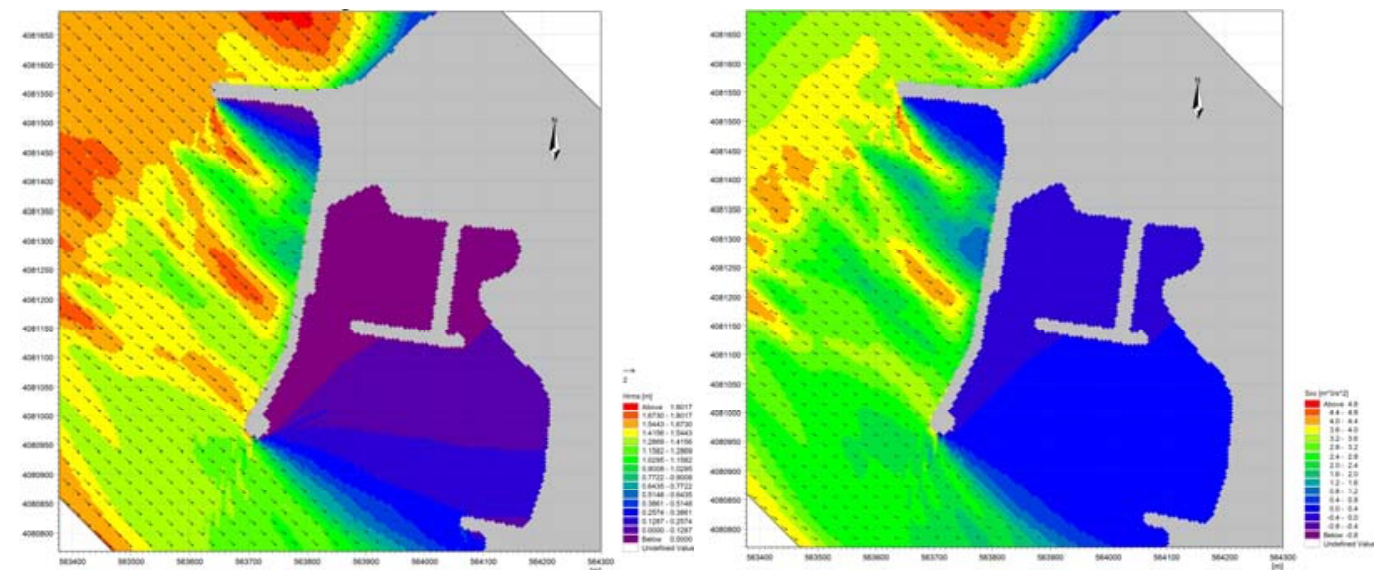

Figure 8. Epure de réfraction de la houle. Figure 9. Champ des contraintes de radiation.
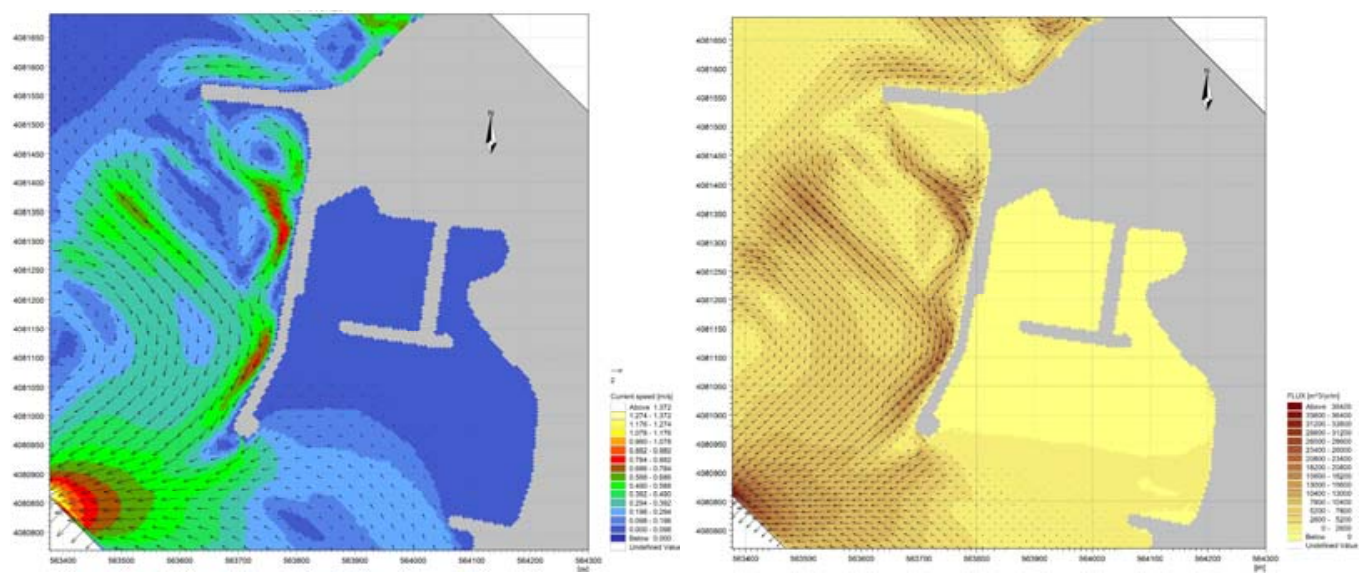

Figure 10. Champ des courants de la houle. Figure 11. Capacité du transport solide.

Les courants littoraux induits par la houle (voir figure 10) sont de direction Nord-Sud et évoluent près du rivage en zone de déferlement en longeant la jetée principale avec une intensité considérable, ce courant perd de son intensité plus en allant vers le sud. La 


\section{XVIèmes Journées Nationales Génie Côtier - Génie Civil \\ Le Havre 2020}

capacité de transport solide (voir figure 11) traduit également la forte agitation notée au niveau des champs de courant. En effet, la zone littorale située au nord du port et le long de la jetée principale est soumise à un transport sédimentaire important, le flux maximum étant noté dans la partie nord. On note la déviation de la trajectoire de ce flux vers le bassin du port. Ce flux perd de son intensité en allant vers le sud.

\subsection{Aménagement du port de cap Djinet}

La recherche d'une nouvelle conception de lutte contre ce phénomène d'ensablement revient à agir directement sur ces courants littoraux générés par la houle au niveau du site d'étude. Dans cet effet, nous avons examiné des variantes d'aménagements de la protection qui consistent en l'introduction d'ouvrages ayant pour objectif l'atténuation de l'action des courants. La variante adéquate (voir figure 12) consiste à prolonger l'épi existant au sud du port en contre jetée, sur une longueur de $300 \mathrm{~m}$ et de $20 \mathrm{~m}$ de largeur. Cette jetée permet d'accélérer la vitesse des courants littoraux au niveau du port et de ce fait, transporter les sédiments au sud en dehors de la zone portuaire. Cette solution doit être optimisée dans le futur, on recommande la projection des épis au nord et sud du port de Cap Djinet.

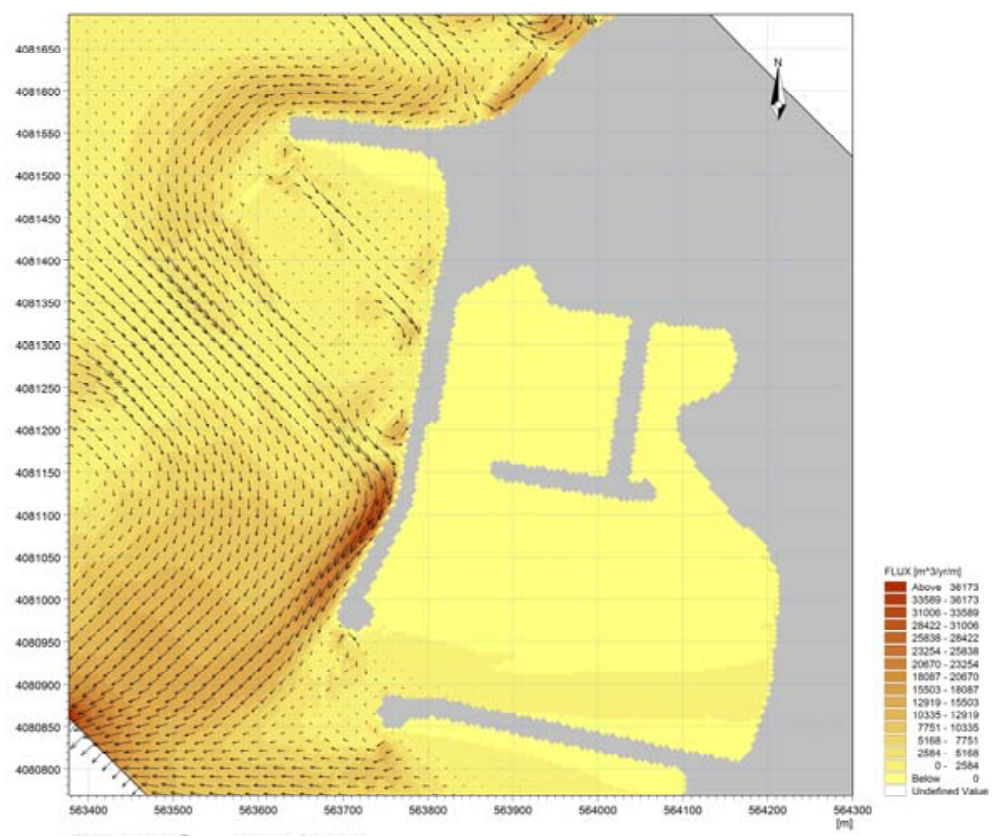

Figure 1. Variante d'aménagement avec une nouvelle jetée projetée sur la côté sud du port Cap Djinet.

\section{Conclusions}

L'examen de l'état initial a permis de mettre en évidence le schéma naturel de la circulation des courants de houles et du transport sédimentaire. Ce dernier se traduit par des courants de dérives littorales qui sont éventuellement liés aux apports importants de 


\section{Thème 2 - Dynamique sédimentaire}

de l'oued Isser. L'analyse des résultats des flux hydro-sédimentaires montre sur le plan technique et économique la variante la plus appropriée pour résoudre la problématique de ce port pour laquelle les champs des courants et du flux sédimentaire sont déviés vers le large et ce, quelle que soit la direction de la houle considérée. Cependant, ce dispositif n'enraye pas définitivement la sédimentation au niveau de la zone d'étude. Il permet par son fonctionnement d'agir sur la périodicité des opérations de dragage d'entretien en augmentant la durée séparant deux opérations successives, ce temps sera d'autant plus important si l'épi nord existant est soulagé périodiquement. Une autre solution technique est essentielle, à savoir l'étude d'aménagement des oueds Isser et Sebaou à travers la réalisation des seuils en gabion avant l'embouchure afin de réduire le transport des apports des sédiments vers la mer.

\section{Remerciements :}

Nos remerciements s'adressent à l'ensemble de notre équipe de recherche, ainsi qu'à tous ceux qui ont organisé les XVI ${ }^{\text {ème }}$ Journées Nationales Génie Côtier - Génie Civil.

\section{Références bibliographiques}

BOUHMADOUCHE M. (2012). Contribution à l'étude géologique et sédimentologique de la grande baie de Zemmouri (Cap-Matifou-Cap Djinet).Thèse de doctorat d'Etat Option Géologie Marine, USTHB, 157 p.

DAHMANI H. (2012). Étude hydro-sédimentaire en domaine bidimensionnel dans un côtier par un progiciel MIKE 21 et LITPACK (cas d'étude du port de Khemisti Wilaya de Tipaza). Mémoire de magister, ENP Alger, pp 89-118.

MIHOUBI M. K., DAHMANI H., KETTAB A., KHODJET KESBA O. (2011). Modélisation numérique de la houle côtière et du transport sédimentaire pour l'étude d'un port de pêche: cas du port de Khemisti. Conférence Méditerranéenne Côtière et Maritime. Edition2, Tanger, Maroc, pp 225-228. https://doi.org/10.5150/cmcm.2011.048

MIHOUBI M. K., DAHMANI H., BELKESSA R. (2012). Simulation numérique du transit sédimentaire d'un aménagement côtier. Journées Nationales Génie Côtier - Génie Civil, Cherbourg, pp 331-338. https://doi.org/10.5150/jngcgc.2012.035-M

MIHOUBI M. K., DAHMANI H. (2015). Numerical modeling of waves and currents by using a hydro sedimentary model, World Academy of Science, Engineering and Technology International Journal of Computer, Electrical, Automation, Control and Information Engineering, Vol. 9, (3), pp 910-917.

VIGUIER J. (2002). Cours de transport sédimentaire maritime. Ecole Nationale des Travaux Publics de l'Etat, SOGREAH Maritime, 16 p. 